dependent transformation that affects its susceptibility to added trypsin. They do not specify how damage is incurred but assume that atrazine and diuron prevent that damage by inhibiting electron transport.

Kyle et al..$^{5}$, on the other hand, make a specific proposal concerning the connection between electron transport and breakdown of $\mathrm{Q}_{\mathrm{B}}$-protein. Working with the green alga Chlamydomonas reinhardii, they demonstrate a correlation between photoinhibition of photosystem II activity and the rate of turnover of $Q_{B}$-protein. When leaves or chloroplasts of higher plants are exposed to supraoptimal light intensities, their photosynthetic activity decreases ${ }^{6}$. This phenomenon is referred to as photoinhibition and is associated with damage to photosystem II. Kyle et al. induce a version of photoinhibition in $C$. reinhardii by exposing cells to a light intensity ten times higher than that encountered during growth. The primary site of photoinhibition is at the reducing side of photosystem II (between photosystem II and plastoquinone) and the degree of photoinhibition is correlated with a loss of binding sites for ${ }^{14} \mathrm{C}$-atrazine, suggesting that molecules of $Q_{B}$-protein have become nonfunctional. Since this form of photoinhibition is exacerbated by conditions which promote reduction of the plastoquinone (PQ) pool (high light intensities and blocking of $\mathrm{PQH}_{2}$ oxidation), Kyle et al. suggest that a high $\mathrm{PQH}_{2} / \mathrm{PQ}$ ratio increases the likelihood that the $Q_{B}$ site will be occupied by the quinone anion $\mathrm{Q}_{B}{ }^{2-}$. Should $\mathrm{Q}_{\mathrm{B}}{ }^{2-}$ interact with $\mathrm{O}_{2}$ generated by photosystem II, highly reactive oxygen radicals would be produced and would selectively damage $\mathrm{Q}_{\mathrm{B}}$-protein by reacting with specific amino acid side chains. Thus, Kyle et al. regard the breakdown of $Q_{B}$-protein as part of the process whereby damaged protein is removed from thylakoids; conversely, the continued synthesis and insertion of $\mathrm{Q}_{\mathrm{B}}$ protein is essential to the process of repair. Ironically, herbicides such as atrazine and diuron reduce the light-induced damage partly by slowing the rate of plastoquinone reduction and partly by occupying the $Q_{B^{-}}$ binding site and preventing the binding of $\mathrm{Q}_{\mathrm{B}}{ }^{2-}$.

Further studies on this system will clearly be of importance for understanding how plants tolerate high light intensities. Of particular interest is the possibility that the synthesis of new $Q_{B}$-protein molecules is somehow regulated by the removal of damaged molecules.

1. Silverthorne, J. \& Ellis, R.J. Biochim. biophys. Acta 607, 319 (1980).

2. Steinback, K.E., McIntosh, L., Bogorad, L. \& Arntzen, C.J. Proc. natn. Acad. Sci. U.S.A. 78,7463 (1981).

3. Vermaas, W.F.J., Arntzen, C.J., Gu, L.-Q. \& Yu, C.-A. Biochim. biophys. Acta 723, 266 (1983).

4. Mattoo, A.K., Hoffman-Falk, H., Marder, J.B. \& Edelman, M. Proc, natn. Acad. Sci. U.S.A. 81, 1380 (1984). 5. Kyle, D.J., Ohad, I. \& Arntzen, C.J. Proc. natn. Acad. Sci. U.S.A. 81, 4070 (1984).

6. Osmond, C.B. Biochim. biophys. Acta 639, 77 (1981).

John Bennett is in the Biology Department, Brookhaven National Laboratory, Associated Universities Inc., Upton, Long Island, New York 11973.
Astronomy

\section{IRAS circulars 14 and 15}

Circular 14 contains 24 IRAS sources in a southern field between right ascension (RA) 10 and $14 \mathrm{~h}$ and between declination (Dec) -40 and $-20 \mathrm{deg}$. All sources shown here are associated with galaxies on the ESO/SRC skyplates; all have larger infrared than visual luminosities. Selection by T. de Jong, University of Amsterdam.

Circular 15 contains a selection of infrared bright northern galaxies with radial velocities larger than $1,000 \mathrm{~km} \mathrm{~s}^{-1}$; they are therefore sufficiently far away that the point source extraction algorithm will estimate the integrated fluxes correctly.

The source name consists of four parts: the letters IRAS to indicate the origin; RA in hours and minutes, seconds omitted; Dec in decimal degrees, multiplied by 10 and then truncated (i.e. 32 deg $42.3 \mathrm{~min} \rightarrow+327)$; an appendix starting with ' $\mathrm{P}$ ' and followed by the number of the circular, this appendix stresses that the data are preliminary. Position is given at equinox 1950.0. Measurements for circular 14 were made between epochs 1983.1 and 1983.9. am, arc min.

\begin{tabular}{|c|c|c|c|c|c|c|}
\hline \multirow{2}{*}{$\begin{array}{l}\text { Source } \\
\text { IRAS }\end{array}$} & RS & Dec & \multicolumn{4}{|c|}{ Flux densities (Janskys) } \\
\hline & $h \min s$ & deg am & $12 \mu \mathrm{m}$ & $25 \mu \mathrm{m}$ & $60 \mu \mathrm{m}$ & $100 \mu \mathrm{m}$ \\
\hline $1021-284 \mathrm{P} 14$ & 102157 & -2828.5 & $<0.2$ & 1.0 & 5.4 & 7.1 \\
\hline $1027-395 \mathrm{P} 14$ & 102720 & -3935.1 & $<0.4$ & 0.4 & 4.1 & 8.1 \\
\hline $1108-282 \mathrm{P} 14$ & 110822 & -2813.7 & $<0.3$ & 0.6 & 3.7 & 5.1 \\
\hline $1116-397 \mathrm{P} 14$ & 111636 & -3943.9 & $<0.2$ & $<0.3$ & 2.5 & 5.5 \\
\hline $1135-325 \mathrm{P} 14$ & 113540 & -3235.1 & $<0.2$ & 0.3 & 3.6 & 6.4 \\
\hline $1140-273 P 14$ & 114050 & $-27 \quad 19.3$ & $<0.2$ & $<0.3$ & 3.0 & 6.0 \\
\hline $1146-330 \mathrm{P} 14$ & 114624 & -3304.0 & $<0.2$ & $<0.2$ & 1.1 & 2.5 \\
\hline $1150-388 \mathrm{P} 14$ & 115040 & $-38 \quad 51.2$ & 0.6 & 2.4 & 39.0 & 55.0 \\
\hline $1203-322 \mathrm{P} 14$ & 120309 & $-32 \quad 16.2$ & $<0.2$ & $<0.2$ & 3.2 & 7.9 \\
\hline $1204-316 P 14$ & $1204 \quad 17$ & $\begin{array}{ll}-31 & 40.3\end{array}$ & 0.3 & 0.8 & 8.1 & 15.0 \\
\hline $1206-364 \mathrm{P} 14$ & 120624 & -3625.5 & $<0.3$ & $<0.5$ & 3.1 & 6.2 \\
\hline $1217-356 \mathrm{P} 14$ & $\begin{array}{lll}12 & 17 & 21\end{array}$ & -3541.1 & $<0.3$ & $<0.3$ & 2.5 & 5.2 \\
\hline $1227-398 \mathrm{P} 14$ & 122700 & -3950.8 & $<0.2$ & $<0.2$ & 3.1 & 3.4 \\
\hline $1228-260 \mathrm{P} 14$ & $12 \quad 2839$ & $\begin{array}{ll}-26 & 00.7\end{array}$ & $<0.2$ & 0.9 & 4.7 & 8.3 \\
\hline $1242-201 \mathrm{P} 14$ & $12 \quad 42 \quad 12$ & -2009.0 & $<0.2$ & $<0.4$ & 3.1 & 7.4 \\
\hline $1250-271 \mathrm{P} 14$ & 125029 & -2711.5 & $<0.3$ & 0.7 & 5.5 & 9.0 \\
\hline $1255-294 \mathrm{P} 14$ & 125502 & $\begin{array}{ll}-29 & 29.8\end{array}$ & $<0.4$ & 1.0 & 7.0 & 10.2 \\
\hline $1300-236 \mathrm{P} 14$ & 130011 & $-23 \quad 39.2$ & $<0.4$ & 0.9 & 15.8 & 20.0 \\
\hline $1304-335 P 14$ & 130422 & -3335.9 & $<0.3$ & 0.6 & 5.3 & 9.2 \\
\hline $1318-345 \mathrm{P} 14$ & 131805 & -3434.6 & $<0.2$ & 0.4 & 3.0 & 5.2 \\
\hline $1318-314 \mathrm{P} 14$ & 131807 & -3128.7 & $<0.2$ & $<0.2$ & 1.7 & 3.7 \\
\hline $1319-394 \mathrm{P} 14$ & $\begin{array}{lll}13 & 19 & 45\end{array}$ & -3928.4 & $<0.2$ & 0.5 & 2.9 & 3.5 \\
\hline $1328-324 \mathrm{P} 14$ & $13 \quad 2835$ & $\begin{array}{ll}-32 & 29.2\end{array}$ & $<0.2$ & $<0.3$ & 1.8 & 3.6 \\
\hline $1345-299 \mathrm{P} 14$ & 134529 & -2957.0 & $<0.2$ & $<0.3$ & 2.5 & 3.6 \\
\hline $0007+286 \mathrm{P} 15$ & $\begin{array}{lll}00 & 07 & 19\end{array}$ & +2538.8 & 0.5 & 1.2 & 10.2 & 19.6 \\
\hline $0610+783 \mathrm{P} 15$ & $06 \quad 1040$ & +7822.5 & 6.5 & 18.5 & 171 & 260 \\
\hline $0705+188 \mathrm{P} 15$ & $0705 \quad 25$ & +1851.6 & 0.6 & 2.2 & 22 & 40 \\
\hline $0710+858 \mathrm{P} 15$ & $07 \quad 1016$ & +8550.9 & 0.6 & 1.2 & 12.9 & 36 \\
\hline $0733+353 P 15$ & 073340 & +3521.2 & 0.5 & 1.0 & 9.7 & 16.5 \\
\hline $0835+259 \mathrm{P} 15$ & 083525 & +2555.8 & $<0.3$ & 1.9 & 28 & 35 \\
\hline $0842+742 \mathrm{P} 15$ & $08 \quad 42 \quad 33$ & +7416.9 & 0.8 & 2.5 & 18.2 & 33 \\
\hline $0910+403$ P15 & $09 \quad 1054$ & $+40 \quad 19.2$ & 0.6 & 1.6 & 9.7 & 16.5 \\
\hline $0914+422 \mathrm{P} 15$ & $09 \quad 14 \quad 10$ & +4212.5 & 0.8 & 3.3 & 26 & 35 \\
\hline $0939+320 P 15$ & 093955 & +3204.6 & 0.7 & 1.4 & 13.3 & 29 \\
\hline $0951+018 \mathrm{P} 15$ & 095106 & +0148.9 & 0.5 & 1.1 & 10.7 & 25 \\
\hline $0958+559 \mathrm{P} 15$ & $0958 \quad 35$ & +55 55.3 & 1.4 & 2.2 & 49 & 110 \\
\hline $1012+736 \mathrm{P} 15$ & 101239 & +7339.0 & 0.6 & 0.7 & 7.4 & 34 \\
\hline $1013+213 \mathrm{P} 15$ & $10 \quad 13 \quad 48$ & +2122.4 & 0.6 & 1.1 & 10.3 & 22 \\
\hline $1020+201 P 15$ & 102047 & +2007.1 & 0.7 & 1.9 & 8.9 & 21 \\
\hline $1035+537 \mathrm{P} 15$ & 103540 & +5345.9 & 1.4 & 5.0 & 38 & 52 \\
\hline $1049+232$ P 15 & 104953 & +2312.0 & 0.7 & 1.4 & 13.0 & 25 \\
\hline $1100+282 \mathrm{P} 15$ & 110027 & +2814.5 & 1.1 & 4.0 & 21 & 41 \\
\hline $1120+168 \mathrm{P} 15$ & 112017 & +1651.8 & 0.6 & 0.9 & 8.4 & 24 \\
\hline $1124+571 \mathrm{P} 15$ & 112443 & +5709.1 & 1.1 & 1.6 & 15.8 & 37 \\
\hline $1146+489 \mathrm{P} 15$ & 114601 & +4859.3 & 0.9 & 1.2 & 14.9 & 43 \\
\hline $1211+548$ P 15 & 121142 & +5448.1 & 1.0 & 4.7 & 27 & 32 \\
\hline $1308+373 \mathrm{P} 15$ & $\begin{array}{lll}13 & 08 & 37\end{array}$ & +3719.5 & 0.8 & 1.2 & 21 & 73 \\
\hline $1330+630 P 15$ & $13 \quad 3027$ & +6301.3 & 0.3 & 1.0 & 7.9 & 17.5 \\
\hline $1359+595 \mathrm{P} 15$ & 135909 & +5934.2 & 0.6 & 1.8 & 11.7 & 25 \\
\hline $1430+581 \mathrm{P} 15$ & 143038 & +5808.3 & 0.7 & 0.9 & 9.2 & 32 \\
\hline $1534+167$ P15 & $15 \quad 34 \quad 14$ & +1646.2 & 0.7 & 0.9 & 9.6 & 27 \\
\hline $1809+149 \mathrm{P} 15$ & 180935 & +1458.0 & 1.0 & 1.8 & 16.3 & 35 \\
\hline $1821+745 \mathrm{P} 15$ & $1821 \quad 13$ & +7432.2 & 0.8 & 1.1 & 11.4 & 39 \\
\hline $2026+255 \mathrm{P} 15$ & 202627 & +2533.9 & 0.6 & 1.1 & 12.5 & 21 \\
\hline $2300+086 \mathrm{P} 15$ & $2300 \quad 45$ & +0836.3 & 1.4 & 5.8 & 30 & 44 \\
\hline $2302+120 P 15$ & $2302 \quad 26$ & +1203.1 & 0.8 & 3.5 & 13.5 & 31 \\
\hline $2312+042 \mathrm{P} 15$ & $23 \quad 12 \quad 11$ & +0415.6 & 0.9 & 1.7 & 21 & 50 \\
\hline $2317+169 \mathrm{P} 15$ & 231760 & +1657.1 & 0.6 & 1.1 & 10.1 & 24 \\
\hline
\end{tabular}

